\title{
Matter and pseudoscalar densities in lattice QCD
}

\author{
C. Alexandrou \\ Department of Physics, University of Cyprus, P.O. Box 20537, CY-1678 Nicosia, Cyprus \\ $\mathrm{Ph}$. de Forcrand \\ Institute for Theoretical Physics, ETH Hönggerberg, CH-8093 Zürich, Switzerland \\ and CERN, Theory Division, CH-1211 Geneva 23, Switzerland \\ A. Tsapalis \\ Department of Physics, University of Cyprus, P.O. Box 20537, CY-1678 Nicosia, Cyprus
}

(Received 8 July 2003; published 28 October 2003)

\begin{abstract}
The matter and the pseudoscalar densities inside a hadron are calculated via gauge-invariant equal-time correlation functions. A comparison is made between the charge and the matter density distributions for the pion, the rho, the nucleon and the $\Delta^{+}$within the quenched theory, and with two flavors of dynamical quarks.
\end{abstract}

DOI: 10.1103/PhysRevD.68.074504

PACS number(s): 11.15.Ha, 12.38.Aw, 12.38.Gc, 14.70.Dj

\section{INTRODUCTION}

The one-body matter density distribution plays an important role in our understanding of nuclear structure. In nuclei it determines the intranuclear distribution which includes both protons and neutrons, unlike the charge density which determines the proton distribution. It requires hadronic probes and for this reason it is not as accurately determined as the charge distribution. However at new experimental facilities such as RIKEN in Japan there are plans to improve the measurements of the matter density distribution using proton beams [1]. Similarly matter density distributions of individual hadrons are determined in hadron proton collisions [2]. From the analysis of elastic differential cross sections for $\pi^{ \pm} p$ and $K^{ \pm} p$, the radii of these mesons can be extracted within certain assumptions such as the eikonal approximation and the exponentiation of the $S$ matrix [3].

In this work we study hadron matter densities using gauge invariant correlators calculated in lattice QCD. This is an extension of our previous work [4] on hadron charge density distributions. In addition to the matter density distribution we present results for the pseudoscalar density and compare to bag model predictions. Both quantities are evaluated in quenched QCD and with two flavors of dynamical quarks. To test our lattice procedure we perform a more detailed analysis of the relevant correlation functions as compared to what was done in Ref. [4] extending it also to the matter and pseudoscalar densities. By comparing the matter and charge density distributions one can draw important phenomenological conclusions regarding hadron deformation.

\section{GAUGE INVARIANT CORRELATION FUNCTIONS}

We consider the equal-time correlators $[5,6]$,

$$
C_{\Gamma}^{H}(\mathbf{r}, t)=\int d^{3} r^{\prime}\left\langle H\left|\hat{j}_{\Gamma}^{u}\left(\mathbf{r}^{\prime}, t\right) \hat{j}_{\Gamma}^{d}\left(\mathbf{r}^{\prime}+\mathbf{r}, t\right)\right| H\right\rangle
$$

with the current $\hat{j}_{\Gamma}^{u}(\mathbf{r}, t)$ given by the normal order product $: \bar{u}(\mathbf{r}, t) \Gamma u(\mathbf{r}, t):$. For $\Gamma=\gamma_{0}$ and $\Gamma=\mathbf{1}$ we obtain the charge and matter density distributions respectively. The pseudoscalar density obtained with $\Gamma=\gamma_{5}$ is also evaluated since it provides a useful observable for testing the predictions of the bag model.

The matrix elements for mesons and baryons considered in this work are shown schematically in Fig. 1. In the case of the baryons two relative distances are involved and three current insertions are required. However we may consider integrating over one relative distance to obtain the oneparticle density distribution shown schematically in the lower part of Fig. 1. Baryon matrix elements with three current insertions, one on each of the three quark lines, were computed in the case of the charge density distribution in Ref. [4] and shown, after integration over one relative distance, to reproduce, within statistics, the one-particle density. In this work we will only consider one-particle distributions, and therefore only diagrams with two current insertions such as shown in Fig. 1 are evaluated. In the future, we plan to extend our work to three current insertions, at equal and unequal times.

We also address here an important technical point. On our lattice of time-extent $T$, with the usual anti-periodic boundary conditions for the fermion fields, we insert the currents at Euclidean at the maximal time separation $T / 4$ from the source and from the sink, as indicated in Fig. 1. The current insertions must be separated far enough from the source and the sink to suppress excited hadronic states and non-zero momenta. The latter are not projected out, as often done in other studies, because zero-momentum projection requires a summation over spatial translations of the source or sink. Here, this summation is technically not feasible because it would involve quark propagators from all to all spatial lattice sites. In the following section we will demonstrate that our time separation of $T / 4$ is sufficient to satisfactorily filter out undesired states.

\section{LATTICE TECHNIQUES}

All the results presented in this work have been obtained on lattices of size $16^{3} \times 32$. For the quenched case we use 

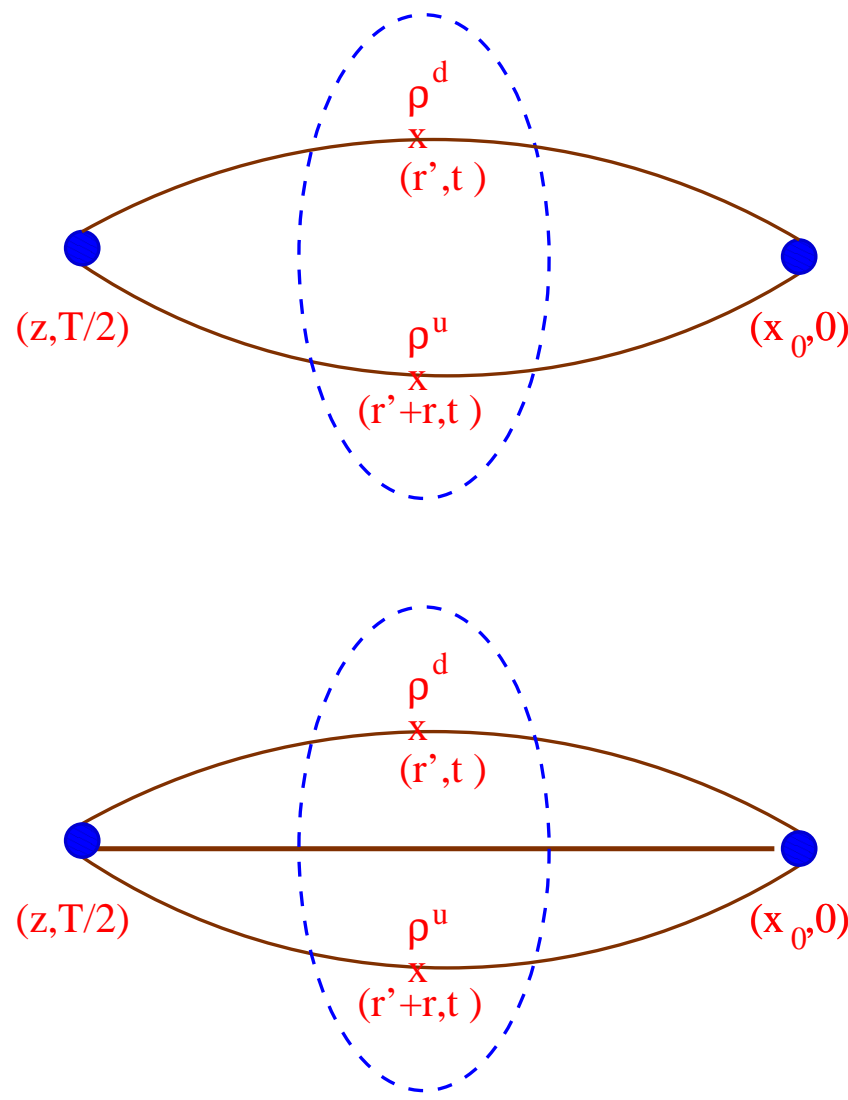

FIG. 1. Equal-time current-current correlator for a meson (upper) and a baryon (lower). $t$ and $T / 2-t$ must be large enough to filter out the hadronic state. $T$ is the time extension of our lattice. We have assumed anti-periodic boundary conditions in the temporal direction in drawing this figure.

220 NERSC [7] configurations generated at $\beta=6.0$, and for the unquenched case we analyze two $\kappa$ values using for each 100 SESAM configurations [8] simulated at $\beta=5.6$ with two degenerate quark flavors. The physical volume of the lattice in the quenched and in the unquenched case is approximately the same.

Let us first demonstrate that the time extent of our lattice is large enough to isolate the hadron ground state of zero momentum. For this study we analyzed 56 quenched configurations at $\kappa=0.153$. The first check is to evaluate the mass of the particles via the two point correlator, $C(t)$, using different sources. For this comparison we use: (i) a local source with anti-periodic boundary conditions in the temporal direction, (ii) a local source with Dirichlet boundary conditions in the temporal direction, (iii) Wuppertal smearing of the source and (iv) a wall source which projects out the zero momentum quark propagator. In the latter case we Coulomb gauge fix the configuration at the time slice of the source. Employing Dirichlet boundary conditions allow us to utilize the whole time extent of our lattice instead of using half as it is done for anti-periodic boundary conditions in the rest of the cases. When using Dirichlet boundary conditions the source is placed at the second time slice, the sink at the last but one and the current insertions at various intermediate time separations, $t$, from the source. In all cases we use a

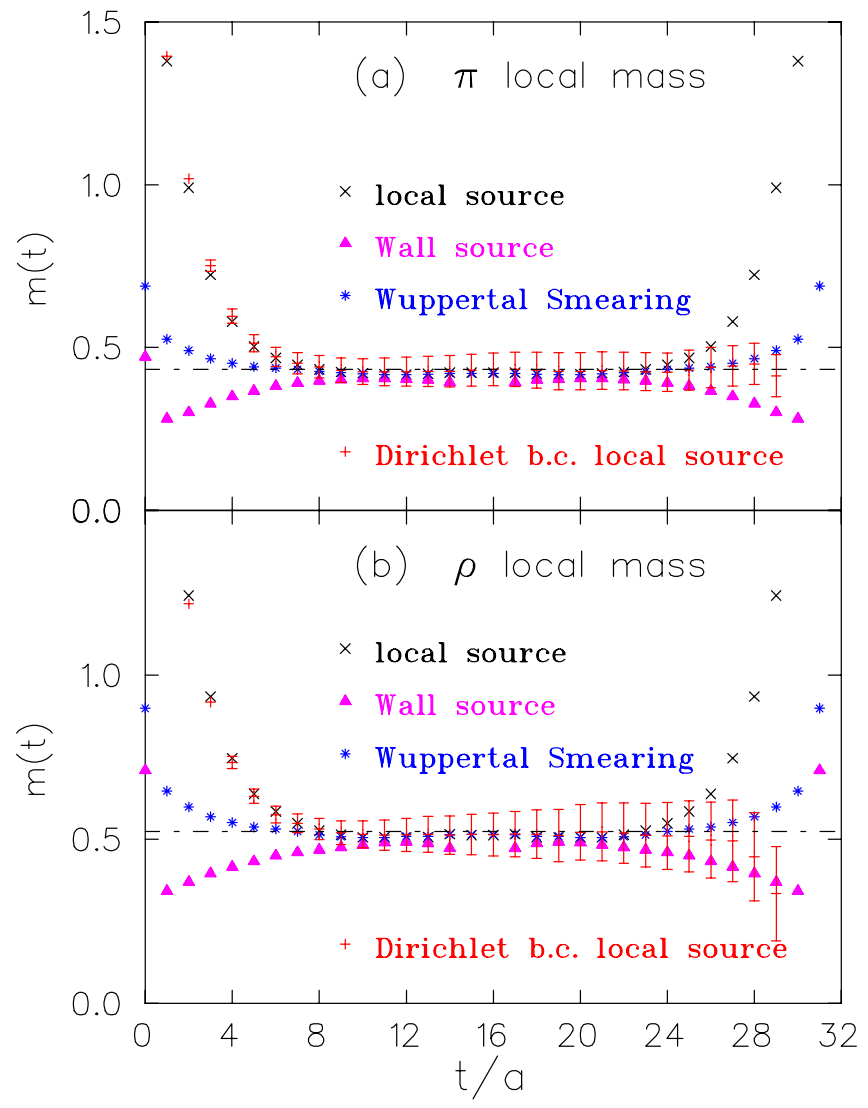

FIG. 2. The local mass, $m(t)$, at $\kappa=0.153$ (a) for the pion and (b) for the rho is shown versus the time separation from the source in lattice units, for a local source with anti-periodic boundary conditions ( $X$ 's), a local source with Dirichlet boundary conditions (crosses), a smeared source (stars) and a wall source (filled triangle). The dashed line shows the plateau value obtained by fitting the data coming from Dirichlet boundary conditions.

local sink. The results for the effective mass, $m(t)$, for the pion and the rho are shown in Fig. 2. As expected Wuppertal smearing of the source produces the earliest plateau. Local sources reach the same plateau value for time separations $t$ $\geqslant 8 a$ from the source, where $a$ is the lattice spacing. Using a wall source does not improve projection to the ground state. In fact the effective mass still deviates at $t=8 a$ and only converges to the plateau value at time separations $t \geqslant 10 a$. For testing the mass plateaus using two point hadron correlators, we have summed over the sink spatial volume which projects to the zero momentum state in the standard way. Had we not performed the sum over the sink spatial volume the errors on the effective mass would have be much larger and the comparison of different sources would have been less meaningful. As we have already pointed out summing over the spatial volume of the source is not possible for the density distributions since it would require the all-to-all propagator. Therefore it is crucial to check that higher momenta are sufficiently suppressed for the evaluation of the density distributions.

In Fig. 3 we show the results for the charge density distribution at different time slices for the pion and the rho, obtained using Dirichlet boundary conditions which allow us 


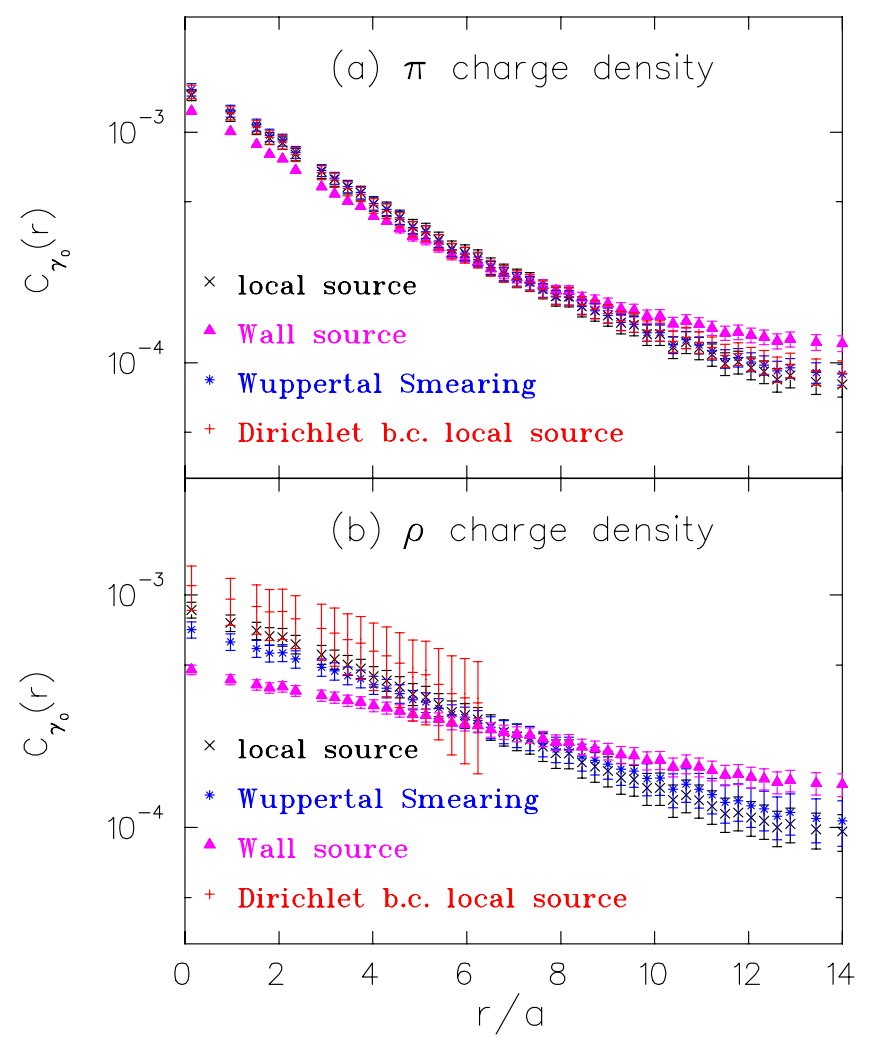

FIG. 3. The charge density distribution at $\kappa=0.153$ (a) for the pion and (b) for the rho is shown versus the radius in lattice units when the current couples to the quark at time separation from the source of $t=8 a$ with anti-periodic boundary conditions and of $t$ $=14 a$ for Dirichlet boundary conditions. In the case of the rho distribution the errors on the data obtained with Dirichlet boundary conditions are omitted for distances greater than $r / a=6$ for clarity. The notation is as in Fig. 2.

to probe larger time separations. Here, in contrast to the twopoint correlator, $C(t)$, the source and the sink are treated in the same way. For all the plots showing the charge density distribution as a function of $r$ we have averaged the lattice results over bins of size $0.28 a$. As it can be seen the results for the pion are the same at quarter lattice time separation $t$ $=8 a$ and at $t=14 a$. We also have agreement between the results obtained with local and smeared sources. For the rho the comparison is more difficult since the results using Dirichlet boundary conditions are more noisy than for the pion. Within our statistics the charge density distributions obtained at time separation $t=8 a$ and $t=14 a$ are the same. Also, apart from small deviations at short distances, there is agreement of the results obtained using Wuppertal smeared and local sources. This means that any contamination from high momentum states is small and, within our statistical uncertainties, it is permissible to use anti-periodic boundary conditions with current insertions at $t=8 a$. This is fortunate, since it allows us to analyze standard full QCD configurations. It is also advantageous since the gauge noise is far less for time separations $8 a$. Using a wall source and sink produces a correlator which is in disagreement with the rest at time separation $t=8 a$. This means that there is still a sizable contamination from excited states, which is not surprising

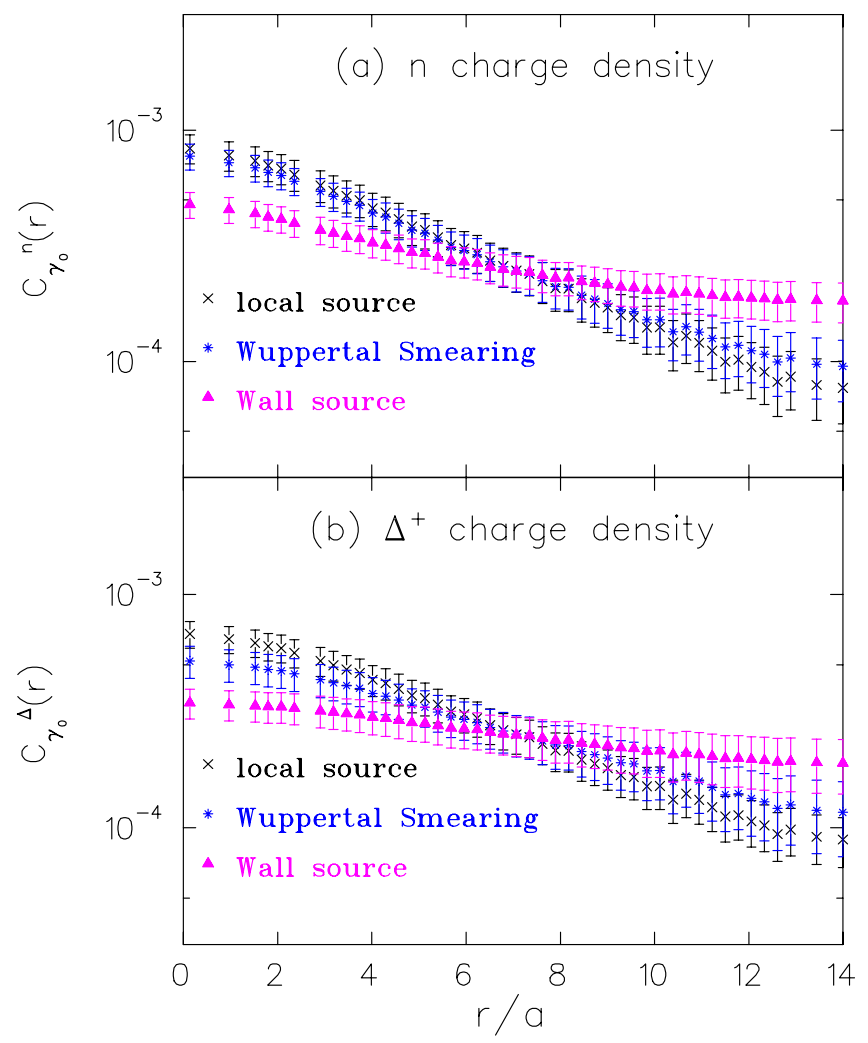

FIG. 4 . The charge density distributions at $\kappa=0.153$ versus the radius in lattice units (a) for the nucleon and (b) for the $\Delta^{+}$using a local source and sink with anti-periodic boundary conditions ( $X$ 's), for a smeared source and sink (stars) and for a wall source and sink (filled triangle). Data obtained using Dirichlet boundary conditions are too noisy and they are not shown.

given that the effective mass for this source has not completely converged at $t=8 \mathrm{a}$. In contrast to the other sources, where the individual quark propagators can carry non-zero momentum, wall sources project on zero momentum for each quark propagator. If the time evolution is too short to filter the ground state then the quarks carry lower momentum than in the exact ground state. This would explain the fact that, for a wall source, the charge density distribution is still broader than the rest at $t=8 a$ : the quarks carrying a smaller momentum lead to a slower decay. This effect is less visible in the pion, which indicates that in the ground state the quark relative momentum is smaller than in the rho. In Fig. 4 we compare the results obtained with the different sources for the nucleon and the $\Delta^{+}$at time separation $t=8 \mathrm{a}$. Data with Dirichlet boundary conditions are not shown since they are too noisy. Again using a wall source and sink produces a correlator that decays more slowly than the rest, showing that projection to the ground state is less effective when the initial and final states are constructed with zero momentum quarks. On the other hand, there is agreement between results obtained with smeared and local sources apart from small deviations at short distances for the $\Delta^{+}$channel. The independence of the results from the different interpolating fields indicates that the ground state has been isolated sufficiently well. Therefore for the parameters used here, it is justified to use anti-periodic boundary conditions and local 


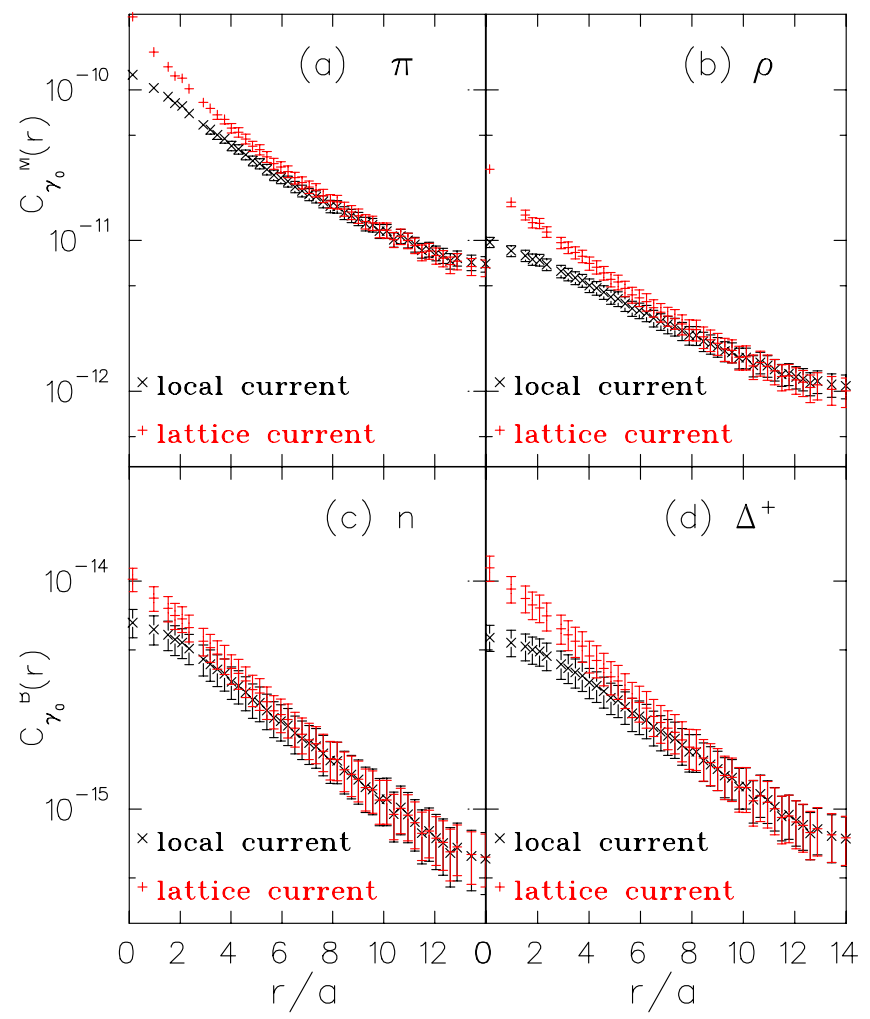

FIG. 5. (a) The pion, (b) the rho, (c) the nucleon and (d) $\Delta^{+}$ charge density distribution versus the radius in lattice units using the local current ( $X$ 's) and the lattice conserved current (crosses).

sources to study the density distributions. Using Dirichlet boundary conditions to increase the time separation between the source and the sink produces results that are more noisy and cannot be distinguished from the ones obtained with anti-periodic boundary conditions.

In this work we use lattices having similar lattice spacing $a$. In order to assess finite lattice spacing effects on these distributions we compare results using the continuum current and the lattice conserved current which differ by $\mathcal{O}(a)$ terms. The lattice conserved current is given by

$$
\begin{aligned}
j_{\mu}(x)= & \sum_{f} Q_{f} \kappa_{f}\left\{\bar{\psi}^{f}(x+\hat{\mu})\left(1+\gamma_{\mu}\right) U^{\mu \dagger}(x) \psi^{f}(x)\right. \\
& \left.-\bar{\psi}^{f}(x)\left(1-\gamma_{\mu}\right) U^{\mu}(x) \psi^{f}(x+\hat{\mu})\right\}
\end{aligned}
$$

and it is symmetrized at lattice site $x . Q_{f}$ is the charge of quark of flavor $f$. The results for the unnormalized density correlator, shown in Fig. 5, demonstrate that finite- $a$ effects lead to the wrong behavior near the origin. The same conclusion was also reached in the study of the density distribution of charmonium where results using the Wilson Dirac operator were compared to those using clover and tadpole improved operators [9]. In the rest of this work, unless otherwise stated, we will normalize the density correlators over the spatial volume and not over their value at the origin because of the potentially large finite $a$ error at the origin. An
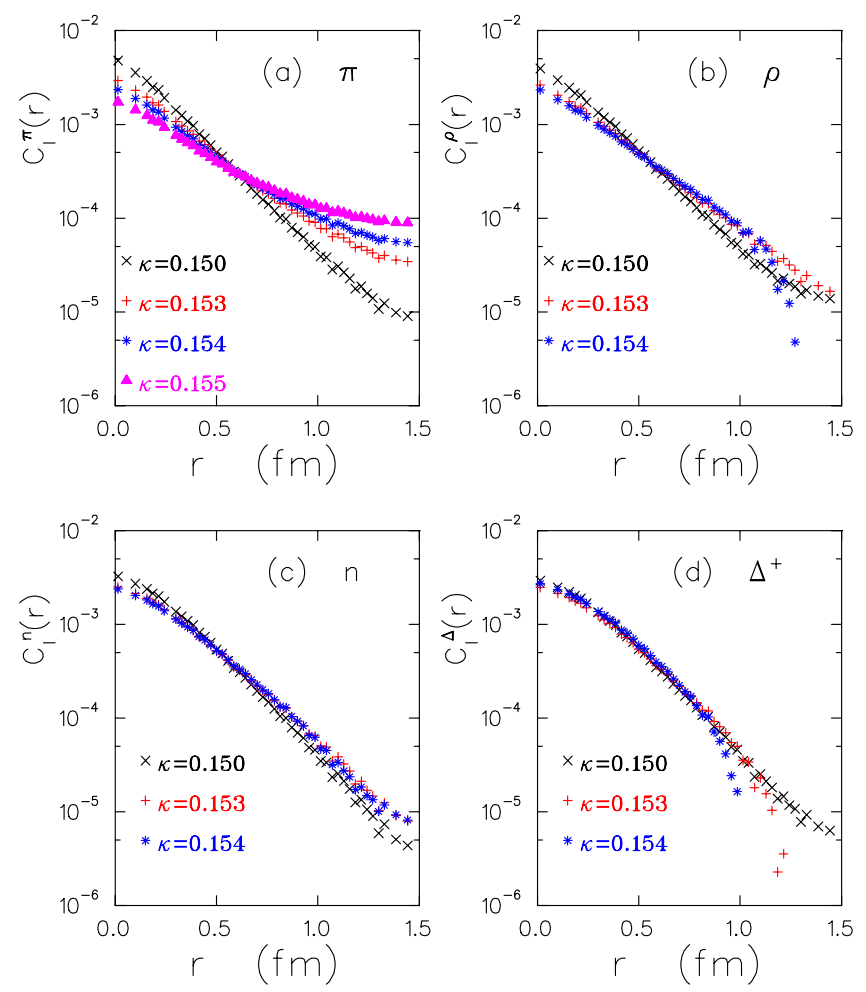

FIG. 6. (a) The pion, (b) the rho, (c) the nucleon and (d) $\Delta^{+}$ matter density distribution versus the radius for $\kappa=0.15$ ( $\left.X^{\prime} \mathrm{s}\right)$, $\kappa=0.153$ (crosses) and $\kappa=0.154$ (stars). For the pion we also include the results for $\kappa=0.155$ (filled triangles). The errors bars are not shown for clarity.

improvement beyond the present work will be to use an $\mathcal{O}(a)$-improved Dirac operator to eliminate finite- $a$ effects at short distances.

\section{MATTER DENSITY}

In this section we present results on the matter density distribution using a local source and a local sink. As discussed in the previous section, our best compromise for the study of the density distribution is to fix the source and the sink for maximum separation at $t_{i}=a$ and $t_{f}=17 a$ and insert $\bar{u} u$ and $\bar{d} d$ operators half-way between at $t-t_{i}=8 a$. By examining the dependence of the matter correlator on the time separation $t-t_{i}$ at which the $\bar{u} u$ and $\bar{d} d$ operators are inserted, as it was done in Sec. III for the charge density, we again conclude that a time interval of $\left|t-t_{i}\right|=\left|t-t_{f}\right|=8 \mathrm{a}$ is sufficient to project to the ground state of the hadrons of interest. In this evaluation we use the same interpolating fields, described in detail in Ref. [4], as for the density correlator, and the Wilson Dirac operator with hopping parameter $\kappa=0.15,0.153,0.154$ and 0.155 . The ratio of the pion mass to the rho mass at these values of $\kappa$ is $0.88,0.84,0.78$ and 0.70 respectively. Using the standard definition of the naive quark mass, $2 m_{q} a=\left(1 / \kappa-1 / \kappa_{c}\right)$, where $\kappa_{c}=0.1571$ is the value of $\kappa$ at which the pion becomes massless, we find $m_{q} \sim 300,170,130$ and $85 \mathrm{MeV}$ respectively. To obtain these values we used the string tension [10] to set the physi- 


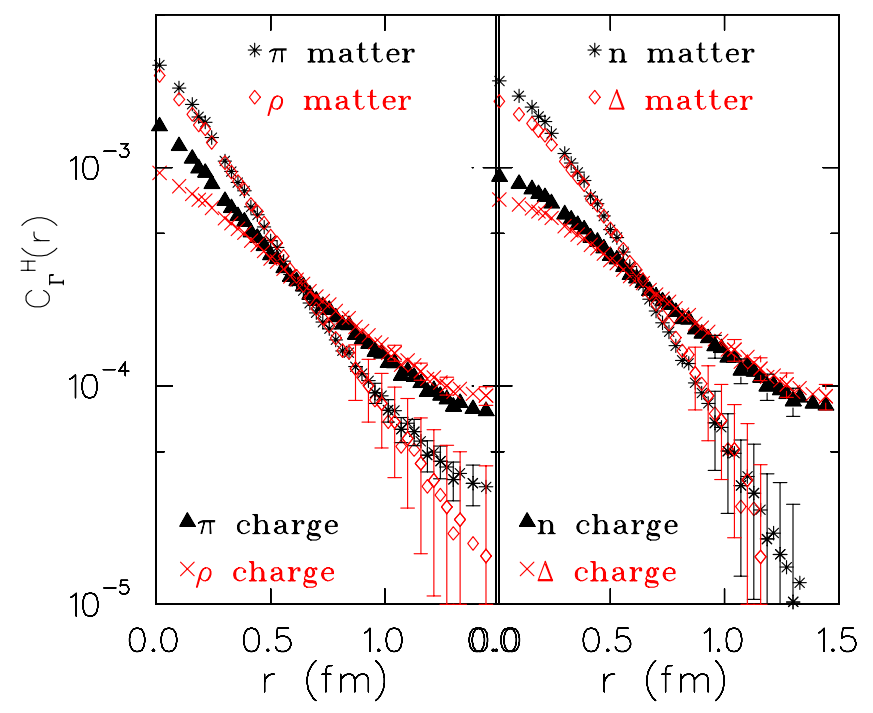

FIG. 7. Comparison of the charge and matter density distributions at $\kappa=0.153$. Left for the pion (filled triangles for the charge) and (stars for the matter) and the rho ( $X$ 's for the charge) and (open rhombus for the matter) distributions. Right for the nucleon (filled triangles for the charge) and (stars for the matter) and the $\Delta^{+}\left(X^{\prime}\right.$ 's for the charge) and (open rhombus for the matter) distributions.

cal scale, which gives for the inverse lattice spacing $a^{-1}$ $\sim 1.94 \mathrm{GeV}(a=0.103 \mathrm{fm})$. This choice makes direct contact to our previous study of charge density distributions [4]. Another possibility is to use the rho (or the nucleon) mass in the chiral limit. Using the rho mass yields $a^{-1}=2.3 \mathrm{GeV}$ $(a=0.087 \mathrm{fm})[11,12]$, with a systematic error coming from the choice of fitting range and chiral extrapolation ansatz of about $10 \%$, which is about twice as large as the statistical error. We note that Ref. [11] observed stability of that scale with respect to an increase of the lattice size. In our discussion of quenched data we will use the value of $a$ determined from the string tension. However, to compare the quenched with the unquenched results we will use the value extracted from the rho mass in the chiral limit, since this determination is applicable both in the quenched and in the unquenched theory. In our discussion of the bag model results we will set the scale using the nucleon mass since, in that case, the nucleon mass is used to fix the bag model parameters.

In Fig. 6 we consider the quark mass dependence of the matter distribution. The pion shows the strongest dependence on the quark mass, in contrast to the charge distribution where the rho showed the largest variation [4]. For quark masses in the range of $300-100 \mathrm{MeV}$ investigated here baryon charge and matter distributions show essentially no variation with the quark mass. However, charge and matter distributions are quite different from each other. The comparison between both is shown in Fig. 7 for $\kappa=0.153$. We observe, between the pion and the rho but not between the nucleon and the $\Delta^{+}$, a larger variation for the charge density as compared to the matter density distribution. In fact the matter density distribution is very similar for the four hadrons considered here. In all cases the matter distribution decays faster than the charge density, an observation consistent with the results of Ref. [13].

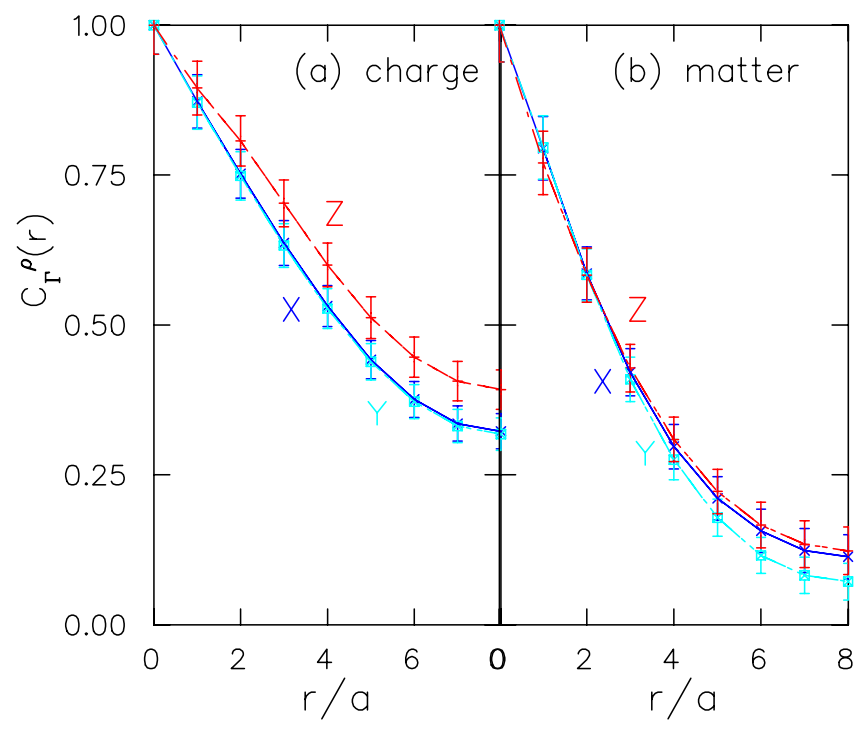

FIG. 8. Rho asymmetry at $\kappa=0.154$ (a) for the charge and (b) for the matter density distributions. The upper curve labeled by $Z$ is $C_{\Gamma}^{\rho}(0,0, z)$ and the lower curves labeled $X$ and $Y$ is $C_{\Gamma}^{\rho}(x, 0,0)$ and $C_{\Gamma}^{\rho}(0, y, 0)$ respectively. The $\mathrm{z}$ axis is along the spin direction of the rho meson.

In Fig. 8 we show the correlators for the spin polarization- 0 state of the rho, for quark separations along the spin axis $(z)$ and perpendicular to it $(x, y)$. We observe a $z-x$ asymmetry in the case of the charge distribution [4] whereas in the case of the matter distribution no statistically significant asymmetry is present. The rho deformation seen in Fig. 8 can be made more quantitative by analyzing the correlators into a dominant $L=0$ and a suppressed $L=2$ state [14]:

$$
\left\langle\rho_{k}(\mathbf{0})\left|\hat{j}_{\gamma_{0}}^{u}(\mathbf{r}) \hat{j}_{\gamma_{0}}^{d}(\mathbf{0})\right| \rho_{k}(\mathbf{0})\right\rangle=f_{0}(r)+\frac{\left(3 x_{k}^{2}-r^{2}\right)}{3 r^{2}} f_{2}(r)
$$

where $\left|\rho_{k}(\mathbf{0})\right\rangle$ is a zero momentum state with polarization $k$. The deformation, $\delta$, determined from the quadrupole moment, can be defined as

$$
\delta \equiv \frac{3}{4} \frac{\left\langle 3 z^{2}-r^{2}\right\rangle}{\left\langle r^{2}\right\rangle} .
$$

Writing for the symmetry axis $R_{3}=\sqrt{\left\langle z^{2}\right\rangle}=R_{T}+\epsilon$ where $R_{T}^{2}=1 / 2\left\langle x^{2}+y^{2}\right\rangle$ and assuming a small symmetry deviation $\epsilon$ we obtain

$$
\delta=\frac{\epsilon}{R_{T}} \sim \frac{R_{3}-R_{T}}{R}
$$

where $R^{2}=1 / 3\left\langle x^{2}+y^{2}+z^{2}\right\rangle$ is the mean square radius. Expanding the hadron wave function in $L=0$ and $L=2$ components as $\psi(\mathbf{r})=\alpha \phi_{0}(r) P_{0}(\cos \theta)+\beta \phi_{2}(r) P_{2}(\cos \theta)$, where $P_{L}$ are Legendre polynomials, we find for the deformation 


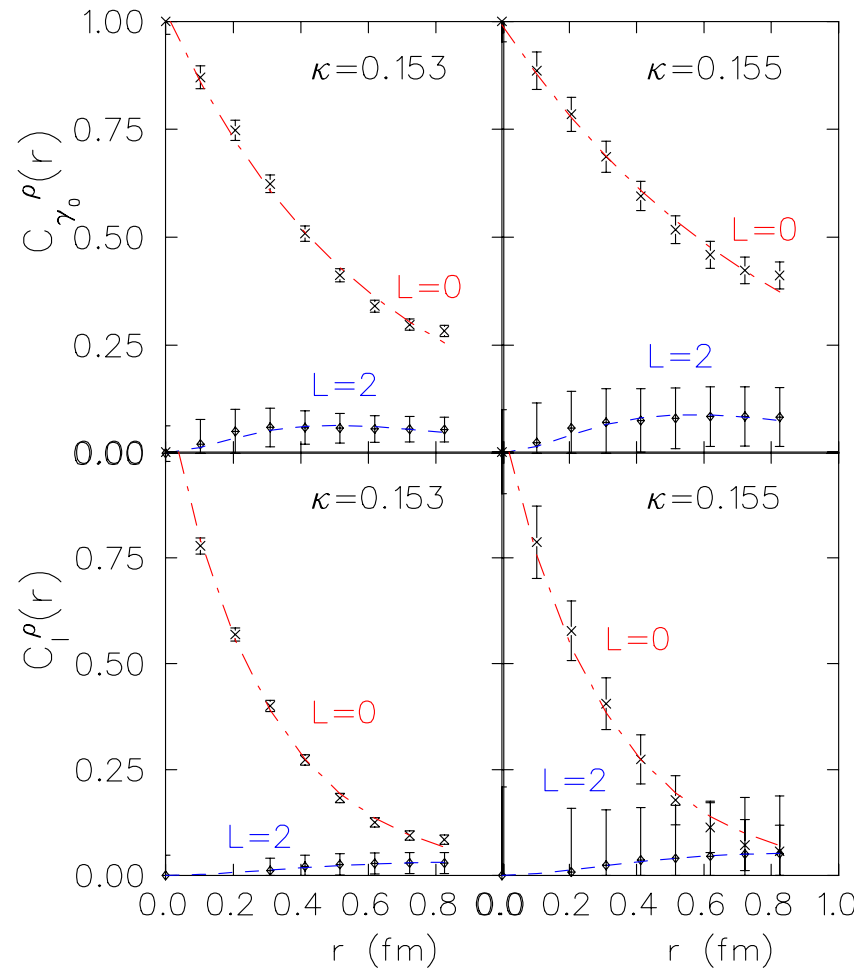

FIG. 9. Decomposition of the density-density correlator, $C_{\gamma_{0}}^{\rho}$, and the matter density correlator, $C_{I}^{\rho}$, for the rho meson into angular momentum part $L=0$ and $L=2$ at $\kappa=0.153$ (left) and $\kappa$ $=0.155$ (right).

$$
\delta=\frac{3}{5} \frac{\beta}{\alpha} \frac{\left\langle\phi_{0}\left|r^{2}\right| \phi_{2}\right\rangle}{\left\langle\phi_{0}\left|r^{2}\right| \phi_{0}\right\rangle}
$$

where we have neglected $\beta^{2}$ terms. Taking $\phi_{0}(r)$ $\sim \exp \left(-m_{0} r\right)$ and $\phi_{2}(r) \sim r^{2} \exp \left(-m_{2} r\right)$, and again neglecting $\beta^{2}$ terms which appear when squaring the wave function to obtain the correlator so that $f_{0}(r)=\alpha^{2} \phi_{0}^{2}(r)$ and $f_{2}(r)$ $=3 \alpha \beta \phi_{0}(r) \phi_{2}(r)$, we fit the resulting expression to the lattice data. As shown in Fig. 9 for two $\kappa$ values, this ansatz provides a good description of the rho charge density distribution. From the fits we find at the four $\kappa$ values that we have analyzed a non-zero ratio $\beta / \alpha \sim 0.02$ with an error of about $60 \%$ which mainly arises from the poor determination of the coefficient of the $L=2$ state. This leads to a deformation of the order of $1 \%$. The errors in extracting $\delta$, within this approach, are large since, in addition to the ratio $\beta / \alpha$, this evaluation also involves $m_{0}^{5}$ and $\left(m_{0}+m_{2}\right)^{7}$ amplifying further the error on $\delta$. A direct determination of the quadrupole moment via Eq. (4) yields $\delta=0.03 \pm 0.01$, with a better control on the errors than the value obtained from the angular decomposition. Unquenching tends to increase this deformation, but the statistical error also increases, so that no definite conclusion regarding the importance of pion cloud contribution can be reached. However, the fact that deformation is seen for the rho meson in the quenched theory casts doubt on models which assume a spherical core and attribute the deformation entirely to the pion cloud [15]. The same analysis for the matter density distribution yields an $L=2$

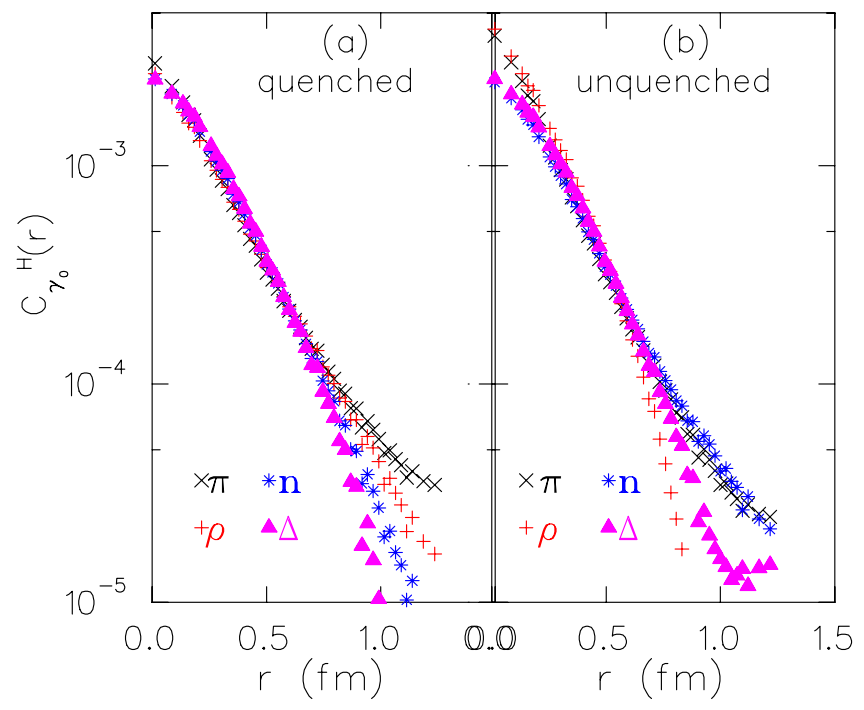

FIG. 10. Comparison of the quenched matter density distribution at $\kappa=0.153$ (left) to the unquenched one at $\kappa=0.156$ (right) for the pion $\left(X\right.$ 's), the rho (crosses), the nucleon (stars) and the $\Delta^{+}$ (filled triangles).

component consistent with zero, as expected from the lack of $z-x$ asymmetry demonstrated in Fig. 8. Also in the unquenched case, the matter density asymmetry remains consistent with zero.

The absence of a measurable deformation in the case of the rho matter distribution has important implications for the mechanism that produces the asymmetry in the rho charge distribution. Since the matter and charge operators have the same non-relativistic limit, this strongly suggests that hadron charge deformation is a relativistic effect. That is particularly interesting since it has strong implications for the validity of various models used in the study of nucleon deformation as well as in the evaluation of transition matrix elements for $\gamma N \rightarrow \Delta$. In a number of these models [16] the quadrupole moments are evaluated in the non-relativistic constituent quark model limit with D-state admixture or with two-body currents where one would expect the same results for the charge and matter distributions. In these models one thus expects that for the rho meson the charge and matter quadrupole moments are the same up to an overall multiplicative factor related to the different electromagnetic and strong coupling constants. The conclusion that the charge deformation is a relativistic effect is confirmed by another study employing heavy quarks on a fine lattice. For these heavy quark systems no signal for charge deformation was obtained [9]. If the pion cloud makes a significant contribution to the charge deformation then an analysis with lighter dynamical quarks should increase the charge deformation but it should also yield a deformation in the matter distribution. Clearly, in view of such important phenomenological implications, it is imperative to improve the lattice results, in particular for the baryons, by using lighter quarks on a larger volume both in the quenched and in the unquenched theory, and determine the amount of deformation in the chiral limit.

In order to investigate the importance of dynamical quarks, we analyze a subset of the SESAM [8] configura- 


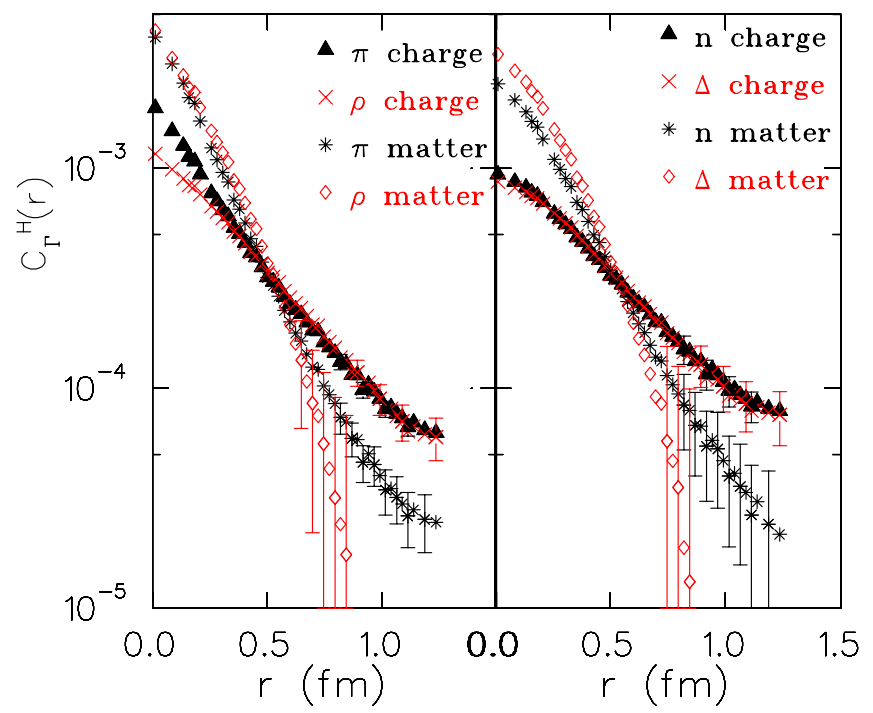

FIG. 11. Comparison of the charge density distribution to the matter distribution for the unquenched theory at $\kappa=0.156$. The notation is the same as that of Fig. 7.

tions. The lattice spacing determined from the rho mass in the chiral limit using the SESAM configurations is $a^{-1}$ $=2.3 \mathrm{GeV}[8]$ which is the same as for the quenched theory at $\beta=6.0$, and therefore the physical volume is the same as in our quenched calculation. As we have already explained, we use this determination of the lattice spacing, which is applicable in both the quenched and the unquenched theory, to compare quenched and unquenched results. For each of the two values of the hopping parameter, $\kappa=0.156$ and 0.157 , we analyzed 100 SESAM configurations. The ratio of the pion to the rho mass is 0.83 at $\kappa=0.156$ and 0.76 at $\kappa$ $=0.157$. These values are close to the quenched mass ratios measured at $\kappa=0.153(0.84)$ and $\kappa=0.154(0.78)$ respectively, allowing us to make pairwise quenched-unquenched comparisons. Such comparison was made for the charge distribution in Ref. [4]. In Fig. 10 we compare the matter distribution for the quenched $(\kappa=0.153)$ and the unquenched $(\kappa=0.156)$ theories. We observe no unquenching effects for the baryons, whereas the unquenched matter density distribution for the pion and rho increases at the origin. Beyond about $0.7 \mathrm{fm}$ the statistical errors, not shown in the figure for clarity, become large especially for the nucleon and the $\Delta^{+}$. In Fig. 11 we compare the unquenched charge and matter density distributions at $\kappa=0.156$. Similar results with larger statistical errors are obtained for the lighter quark mass $(\kappa$ $=0.157$ ), indicating that the mass dependence of these results is very weak. As in the quenched case we observe a faster fall off of the matter density distribution as compared to the charge density distribution. Whereas unquenching increases the rho charge asymmetry (see Fig. 17 of Ref. [4]), it has no effect on the matter density distribution: Fig. 8(b) remains unchanged.

\section{PSEUDOSCALAR DENSITY}

The pseudoscalar density is of theoretical interest, especially because it can serve as an additional observable in our
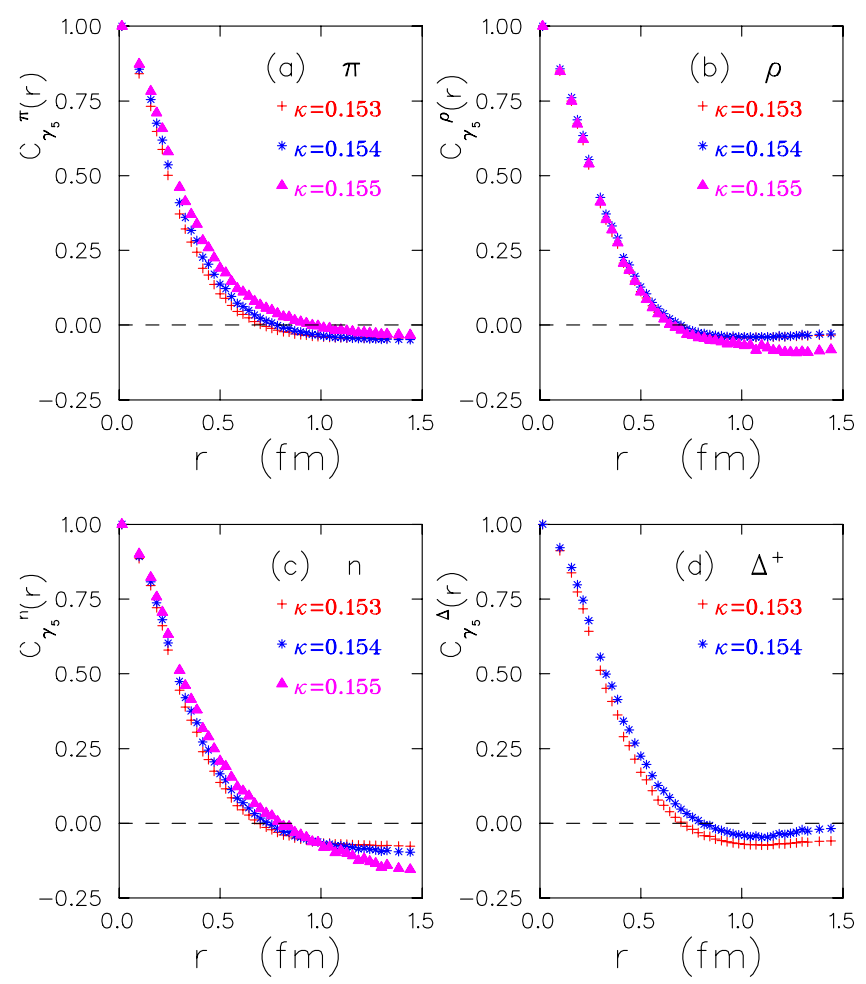

FIG. 12. The pseudoscalar density at $\kappa=0.153,0.154$ and 0.155 for (a) the pion, (b) the rho, (c) the nucleon and the (d) $\Delta^{+}$.

comparison with bag model predictions. The lattice results in the quenched theory for the pion, the rho, the nucleon and the $\Delta^{+}$are shown in Fig. 12 for $m_{\pi} / m_{\rho}=0.84,0.78$ and 0.70 . Again we observe only a weak dependence on the quark mass. To compare with bag model predictions, which will be discussed in the following section, it is best to consider the pseudoscalar density weighted with $r^{2}$. This is shown for both the quenched and the unquenched theory at $\kappa=0.153$ and $\kappa=0.156$ respectively in Fig. 13. A reasonable fit is obtained using an exponential times a polynomial ansatz. We note here that in both the quenched and unquenched theory the long tail of the data and the integral of the fitted ansatz both favor a non-zero integral. In Fig. 14 we show the unquenched pseudoscalar density for the four hadrons under consideration normalized to unity at the origin. We observe that the results are very similar for all hadrons, with almost no dependence on the quark mass. As in the case of the matter distribution dynamical quark effects are small for these values of quark masses. Before leaving this section we must stress that these results on the pseudoscalar density are most useful in showing qualitative features. Since we have used an unimproved Dirac operator which has order $a$ chiral symmetry violations, we expect sizable ultraviolet corrections.

\section{COMPARISON OF LATTICE AND BAG MODEL RESULTS}

It is interesting to compare our lattice results to those obtained in the bag model. We will consider only the lowest mode of the free Dirac field in a spherical bag of radius $R$. 


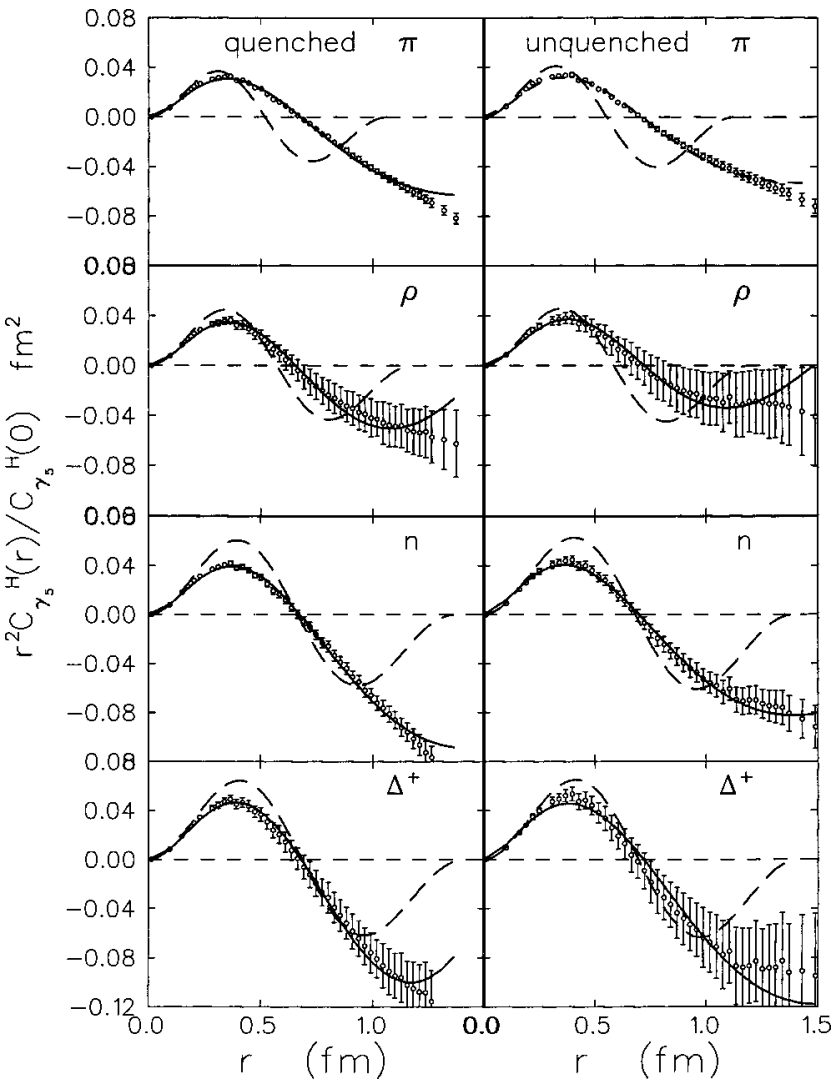

FIG. 13. The quenched (left) and unquenched (right) pseudoscalar density at $\kappa=0.153$ and $\kappa=0.156$ respectively with fits to $\left(a+b x^{2}+c x^{4}\right) e^{-m x}$ (solid line) and bag model results (dashed line).

The radius $R$ is chosen so as to minimize the mass, $M(R)$, of the hadron under consideration. There are four contributions to the mass: the volume term $E_{v}=4 \pi B R^{3} / 3$, the zero point energy, $E_{0}=-Z_{0} / R$, the kinetic energy of the quarks, $E_{Q}$

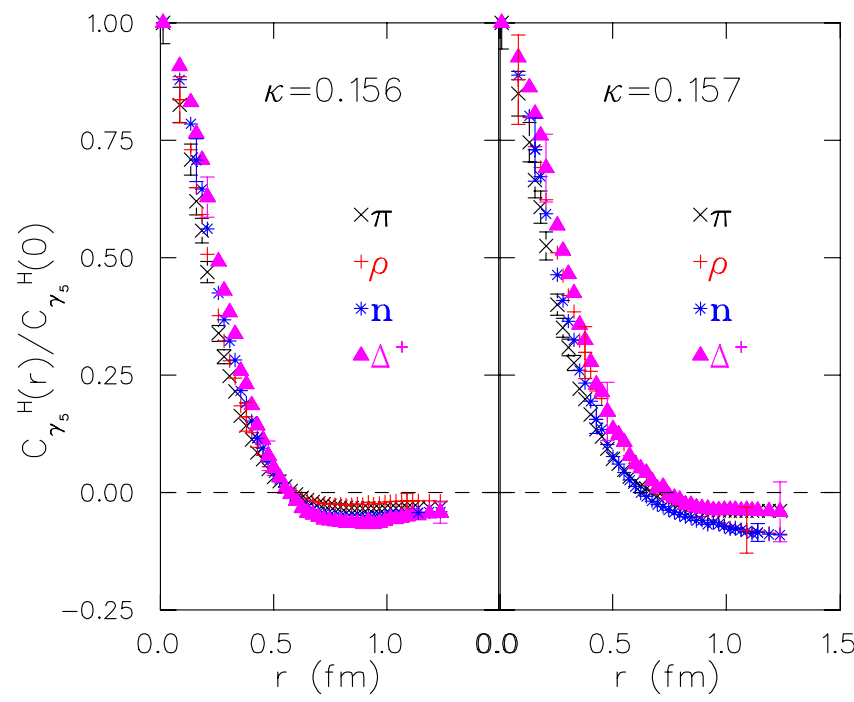

FIG. 14. The unquenched pseudoscalar density at $\kappa=0.156$ (left) and $\kappa=0.157$ (right) for the pion ( $\times$ 's), the rho (crosses), the nucleon (stars) and the $\Delta^{+}$(filled triangles).
TABLE I. Bag model parameters extracted from fitting the masses of the rho, the nucleon and the $\Delta$. The bag radii, $R_{\pi}, R_{\rho}$, $R_{n}$ and $R_{\Delta}$ for the pion, the rho, the nucleon and the $\Delta$ are given in $\mathrm{GeV}^{-1}$ respectively.

\begin{tabular}{cccccccc}
\hline \hline \multicolumn{7}{c}{ Quenched: $\beta=6.0,16^{3} \times 32$} \\
$m_{q}(\mathrm{GeV})$ & $B^{1 / 4}(\mathrm{GeV})$ & $Z_{0}$ & $\alpha_{\text {bag }}$ & $R_{\pi}$ & $R_{\rho}$ & $R_{n}$ & $R_{\Delta}$ \\
\hline 0.174 & 0.209 & 2.274 & 0.169 & 2.71 & 2.99 & 3.48 & 3.59 \\
0.131 & 0.182 & 2.245 & 0.196 & 3.09 & 3.47 & 4.00 & 4.15 \\
0.088 & 0.169 & 2.228 & 0.239 & 3.26 & 3.76 & 4.29 & 4.49 \\
\multicolumn{7}{c}{$N_{f}=2: \beta=5.6,16^{3} \times 32$} \\
$m_{q}(\mathrm{GeV})$ & $B^{1 / 4}(\mathrm{GeV})$ & $Z_{0}$ & $\alpha_{\text {bag }}$ & $R_{\pi}$ & $R_{\rho}$ & $R_{n}$ & $R_{\Delta}$ \\
\hline 0.096 & 0.210 & 2.120 & 0.110 & 2.86 & 3.02 & 3.53 & 3.60 \\
0.076 & 0.195 & 2.096 & 0.136 & 3.06 & 3.28 & 3.80 & 3.89 \\
0.057 & 0.201 & 2.383 & 0.188 & 2.74 & 3.09 & 3.60 & 3.73 \\
0.038 & 0.171 & 2.227 & 0.188 & 3.33 & 3.70 & 4.28 & 4.43 \\
$m_{q}(\mathrm{GeV})$ & $B^{1 / 4}(\mathrm{GeV})$ & $Z_{0}$ & $\alpha_{\text {bag }}$ & $R_{\pi}$ & $R_{\rho}$ & $R_{n}$ & $R_{\Delta}$ \\
\hline 0 & 0.146 & 1.86 & 0.55 & 3.33 & 4.67 & 4.97 & 5.44 \\
\hline \hline
\end{tabular}

$=n_{q} \omega$, and the chromo-magnetic hyper-fine interaction energy calculated to first order in perturbation theory which is proportional to $\alpha_{\mathrm{bag}}$ [17]. $n_{q}$ is the number of quarks in the hadron, $\alpha_{\text {bag }}=g^{2} / 4 \pi$ where $g$ is the strong coupling constant, and $\omega$ is the frequency of the lowest mode given explicitly below. We use two procedures to fix the bag model parameters $B, Z_{0}$ and $\alpha_{\text {bag }}$ : In the first procedure, which we will refer to as procedure $A$, we use the lattice values for the masses of the rho, the nucleon and the $\Delta$ to fix the three parameters using as an input the naive quark mass, $m_{q}$ $=1 / 2 a\left(1 / \kappa-1 / \kappa_{c}\right)$. In Table I we give the bag parameters determined from this procedure. In the quenched case, as the quark mass decreases the bag model parameters nicely approach the parameters determined using the experimental values of the $\omega$ meson, the nucleon and the $\Delta$ and $m_{q}=0$ also included in the last row of Table I. For the unquenched case, this trend is only clear for the two smallest $\kappa$ values (heaviest quarks). For the larger $\kappa$ values this is no longer clear and a possible explanation is finite volume effects known to become important at these small quark masses. A second procedure suggested in Ref. [18], referred to here as procedure $\mathrm{B}$, is to use the bag model parameters as obtained in the last row of Table I and with these parameters fixed, adjust the quark mass so that the lattice results for the mass of the nucleon is reproduced at the given $\kappa$ value. Since we use the nucleon mass to define the quark mass in this way, it is natural for this discussion to use the nucleon mass to set the lattice scale $a$. This gives $a^{-1}=2.04(2) \mathrm{GeV}$ for the quenched case and $a^{-1}=1.88(7)$ for the unquenched theory. The quark mass obtained with procedure B is denoted by $m_{q}^{\text {bag }}$. Both the bag model and the lattice phenomenology are consistent with a linear dependence of the nucleon mass on the quark mass in the regime we explore. Therefore we expect $m_{q} \propto m_{q}^{\text {bag }}$. In Fig. 15 we display the values obtained for $m_{q}^{\text {bag }}$ versus the naive quark mass. The quenched data nicely 


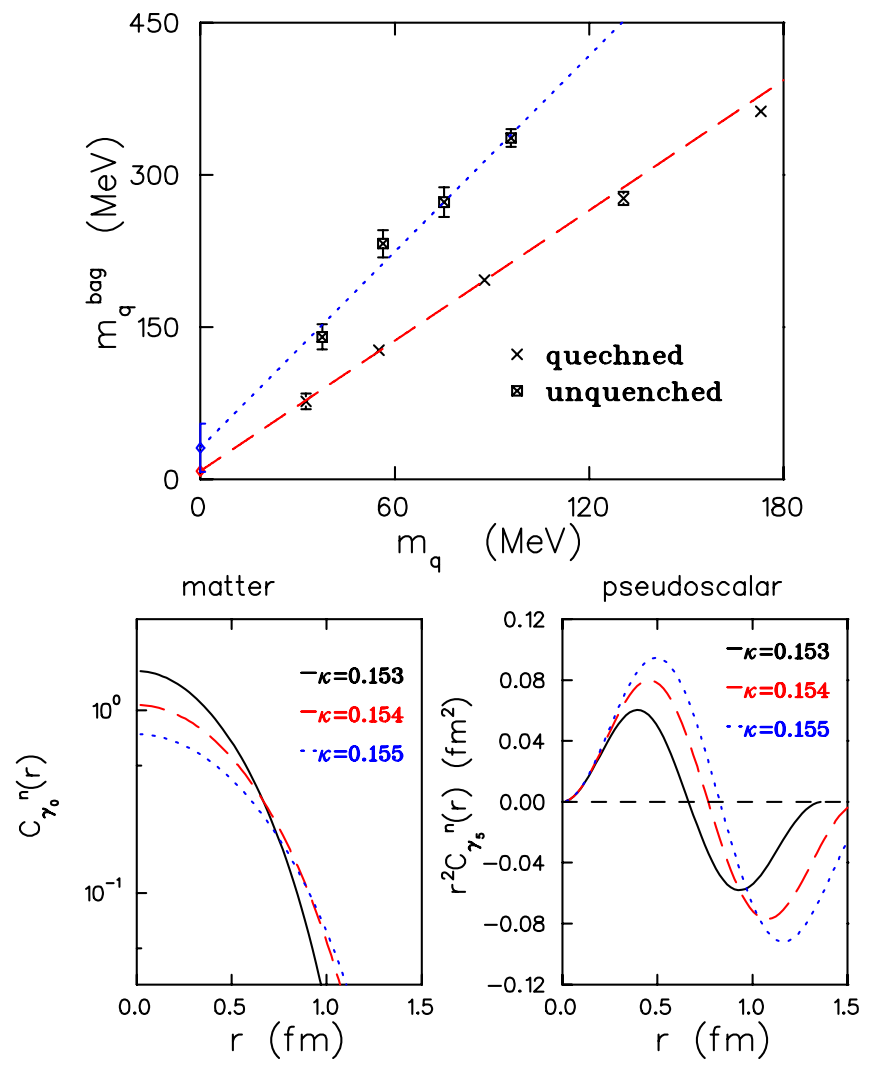

FIG. 15. Upper: The quark mass, $m_{q}^{\text {bag }}$, needed to obtain the lattice result for the nucleon mass versus the naive quark mass $m_{q}$. The best straight line fits to both the quenched (dashed) and unquenched (dotted) case are also displayed. Lower: The mass dependence of the matter (left) and pseudoscalar (right) density distributions for the nucleon within the bag model with parameters fixed using procedure $\mathrm{A}$ at $\kappa=0.153,0.154$ and 0.155 .

fall on a straight line confirming this expectation. We find that $m_{q}^{\mathrm{bag}}$ is about 2.1 times the naive quark mass. Extrapolating to $m_{q}=0$ we obtain $m_{q}^{\mathrm{bag}}=8 \pm 2 \mathrm{MeV}$ reasonably close to the expected zero value, especially since the error does not include uncertainties of the order of $10 \%$ in setting the lattice scale. The same analysis can also be done for the less accurate unquenched data. We find $m_{q}^{\mathrm{bag}} \sim 3 m_{q}$. Using the SESAM results for the nucleon mass at $\kappa$ $=0.156,0.1565,0.157$ and 0.1575 and a linear extrapolation to the chiral limit, we obtain $m_{q}^{\mathrm{bag}}=31 \pm 24 \mathrm{MeV}$ at $m_{q}=0$, again close to zero. In the same figure we also show the bag model results for the matter and pseudoscalar densities calculated with procedure A using the parameters extracted from fitting the quenched data at $\kappa=0.153,0.154$ and $\kappa$ $=0.155$. The bag model results show a stronger dependence on the quark mass in comparison with the corresponding lattice results discussed in the previous sections.

In order to evaluate the various density distributions we expand the quark fields in terms of bag eigenmodes keeping only the lowest mode [18]:

$$
q(\mathbf{r})=\sum_{s, c}\left(b_{s}^{c} \psi_{s}(\mathbf{r})+d_{s}^{c \dagger} \phi_{s}(\mathbf{r})\right)
$$

$$
\begin{aligned}
\psi_{s} & =\left(\begin{array}{c}
i f(r) U_{s} \\
-g(r) \boldsymbol{\sigma} \cdot \hat{\mathbf{r}} U_{s}
\end{array}\right), \quad \phi_{s}(\mathbf{r})=C \gamma_{0} \psi_{s}^{*}(\mathbf{r}) \\
f(r) & =\frac{N(x)}{\sqrt{4 \pi}}\left(\frac{\omega+m}{\omega}\right)^{1 / 2} j_{0}(x r / R) \\
g(r) & =\frac{N(x)}{\sqrt{4 \pi}}\left(\frac{\omega-m}{\omega}\right)^{1 / 2} j_{1}(x r / R)
\end{aligned}
$$

where $U_{s}$ are two component Pauli spinors, $C=\gamma_{2} \gamma_{0}$ is the charge operator, $j_{i}$ are spherical Bessel functions, $m$ is the value of the quark mass which here is either $m_{q}$ or $m_{q}^{\mathrm{bag}}$ and $N(x)$ is fixed by normalizing the eigenmode in the bag. The frequency, $\omega(m, R)$, of the lowest mode is given by $\omega$ $=1 / R\left(x^{2}+m^{2} R^{2}\right)^{1 / 2}$ where $x(m R)$ is the solution of the eigenvalue equation $(1-m R-\omega R) \tan x=x$. The superscript $c$ on the quark annihilation operators $b_{s}^{c}$ and on the anti-quark creation operators $d_{s}^{c \dagger}$ denotes flavor and color quantum numbers. In terms of the lowest eigenmode the density operators are given by

$$
\begin{gathered}
\hat{\rho}_{\gamma_{0}}(\mathbf{r})=\sum_{s, c}\left(b_{s}^{c \dagger} b_{s}^{c}-d_{s}^{c \dagger} d_{s}^{c}\right)\left(f(r)^{2}+g(r)^{2}\right) \\
\hat{\rho}_{I}(\mathbf{r})=\sum_{s, c}\left(b_{s}^{c \dagger} b_{s}^{c}+d_{s}^{c \dagger} d_{s}^{c}\right)\left(f(r)^{2}-g(r)^{2}\right) \\
\hat{\boldsymbol{\rho}}_{\gamma_{5}}(\mathbf{r})=-2 i \sum_{s, s^{\prime}, c}\left(b_{s}^{c \dagger} b_{s^{\prime}}^{c} U_{s}^{\dagger} \boldsymbol{\sigma} \cdot \hat{\mathbf{r}} U_{s^{\prime}}\right. \\
\left.-d_{s}^{c \dagger} d_{s^{\prime}}^{c} U_{s}^{\dagger} \boldsymbol{\sigma}^{*} \cdot \hat{\mathbf{r}} U_{s^{\prime}}\right) f(r) g(r)
\end{gathered}
$$

and the charge, matter and pseudoscalar correlators by the following expressions:

$$
\begin{aligned}
& \left(\begin{array}{ccc}
\pi \mid & & \mid \pi \\
\rho \mid & \hat{j}_{\gamma_{0}}^{u}(\mathbf{r}) \hat{j}_{\gamma_{0}}^{d}\left(\mathbf{r}^{\prime}\right) & \mid \rho \\
n \mid & & \mid n \\
\Delta \mid & \mid \Delta
\end{array}\right)=C\left(\begin{array}{c}
-1 \\
-1 \\
1 \\
1
\end{array}\right)\left(f(r)^{2}+g(r)^{2}\right) . \\
& \left(\begin{array}{ccc}
\pi \mid & & \mid \pi \\
\rho \mid & \hat{j}_{I}^{u}(\mathbf{r}) \hat{j}_{I}^{d}\left(\mathbf{r}^{\prime}\right) & \mid \rho \\
n \mid & \mid n \\
\Delta \mid & \mid \Delta
\end{array}\right)=C^{\prime}\left(\begin{array}{l}
1 \\
1 \\
1 \\
1
\end{array}\right) \begin{array}{c}
\left(f(r)^{2}-g(r)^{2}\right) . \\
\left(f\left(r^{\prime}\right)^{2}-g\left(r^{\prime}\right)^{2}\right)
\end{array} \\
& \left(\begin{array}{ccc}
\pi \mid & & \mid \pi \\
\rho \mid & \hat{j}_{\gamma_{5}}^{u}(\mathbf{r}) \hat{j}_{\gamma_{5}}^{d}\left(\mathbf{r}^{\prime}\right) & \mid \rho \\
n \mid & & \mid n \\
\Delta \mid &
\end{array}\right)=C^{\prime \prime}\left(\begin{array}{c}
1 \\
-1 / 3 \\
2 / 3 \\
-2 / 3
\end{array}\right) 4 \hat{\mathbf{r}} \cdot \hat{\mathbf{r}}^{\prime} f(r) g(r)
\end{aligned}
$$


with constants $C, C^{\prime}$ and $C^{\prime \prime}$ independent of the hadron state. The minus sign for mesons in the charge correlator arises because in the density operator the term involving anti-quarks comes with a negative sign. This term is positive in the case of the matter density giving the same sign for mesons and baryons. This shows explicitly that the difference between charge and matter density distributions is due to the opposite sign of the lower components of the Dirac spinor, which it is a relativistic effect.

In the case of the pseudoscalar correlator given by Eq. (13) the results were obtained by averaging over spin projections of the physical states i.e. for the rho we averaged over the states with $J_{z}= \pm 1$ and $J_{z}=0$. In order to understand the signs for the pseudoscalar density consider the pion where the spin of the quark and the anti-quark are opposite. Since $|\pi\rangle=1 / \sqrt{2} \Sigma_{s}\left(b_{+}^{u \dagger} d_{-}^{d \dagger}-b_{-}^{u}{ }^{\dagger} d_{+}^{d \dagger}\right)|0\rangle$, we have from Eq. (10) a contribution of the form $\pm \boldsymbol{\sigma} \cdot \hat{\mathbf{r}} U_{ \pm} U_{\mp} \boldsymbol{\sigma}^{*} \cdot \hat{\mathbf{r}}^{\prime} U_{-}$yielding the result $-\hat{\mathbf{r}} \cdot \hat{\mathbf{r}}^{\prime}$ and thus an overall positive sign. The rho state with $J_{z}=0$ is orthogonal to the pion resulting in the opposite sign. Similar considerations lead to the opposite sign between the nucleon and the $\Delta^{+}$.

In Fig. 16 we compare the lattice results for the charge and matter density distributions with the bag model results from Eqs. (11) and (12). The dashed lines show the results obtained with procedure A. The dotted lines are the bag model results obtained within procedure B. Both procedures fail to reproduce the correct radial dependence of the charge distribution. The results obtained using procedure A provide an overall better description in the case of the baryon matter distribution. To make the comparison quantitative, we evaluate the root mean square radius, which provides a measure of the width of the distributions. In the bag model one usually computes the expectation value of $\mathbf{r}^{2}$ for the lowest eigenmode. In our previous study of the charge density distribution [4] we used the quark model definition for the charge radius, which for mesons is given by

$$
\begin{aligned}
\left\langle r_{\mathrm{ch}}^{2}\right\rangle & =\sum_{q} e_{q}\left\langle\left(\mathbf{r}_{q}-\mathbf{R}_{\mathrm{cm}}\right)^{2}\right\rangle \\
= & \frac{\sum_{q} e_{q} \int d^{3} r(\mathbf{r} / 2)^{2} C_{\gamma_{0}}(\mathbf{r})}{\int d^{3} r C_{\gamma_{0}}(\mathbf{r})}
\end{aligned}
$$

where $\mathbf{R}_{\mathrm{cm}}$ is the coordinate of the center of mass and $e_{q}$ is the electric charge of the quarks. A corresponding definition for baryons can only be used if one knows the charge density distribution in terms of the two relative coordinates which requires the evaluation of three current correlation functions. Here we only evaluate the one-density baryon charge distribution and therefore the quark model definition cannot be applied. For simplicity and for direct comparison with the bag model radial width we calculate for both mesons and baryons

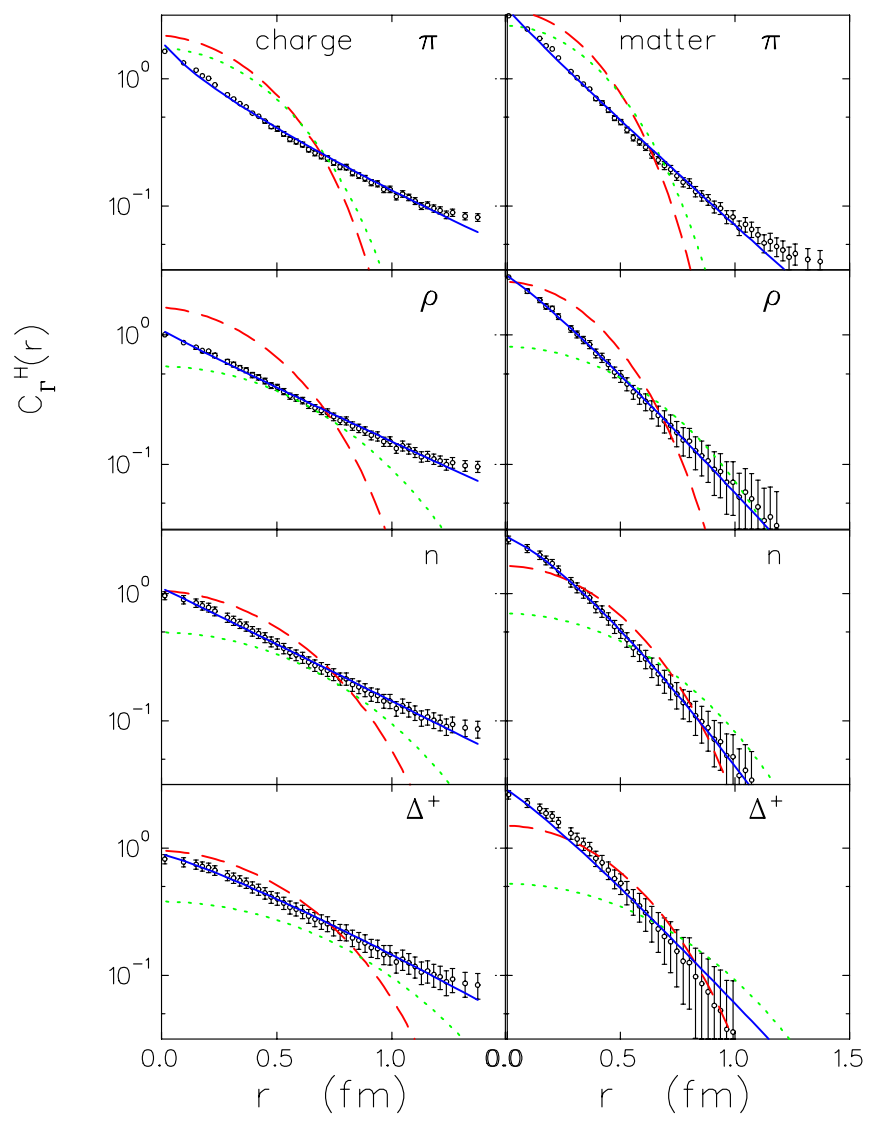

FIG. 16. Comparison of quenched charge (left) and matter distribution (right) at $\kappa=0.153$ with bag model results. The dashed line is obtained by using procedure A as described in the text and the dotted line by using procedure B. The solid line is a fit to $a \exp \left(-m r^{\alpha}\right)$.

$$
\left\langle r^{2}\right\rangle=\frac{1}{2} \frac{\int d^{3} r \mathbf{r}^{2} C_{\Gamma}(\mathbf{r})}{\int d^{3} r C_{\Gamma}(\mathbf{r})},
$$

where for $\Gamma=\gamma_{0}$ we obtain the charge mean square radius, $r_{c h}^{2}$, and for $\Gamma=I$ the matter mean square radius, $r_{m}^{2}$. For degenerate quarks and for the case where the meson wave function is a product of single particle radial wave functions like in the bag model, the factor of $1 / 2$ corrects for summing over the charge root mean square radius of each quark as it is done in Eq. (15) in using the relative quark distance square.

The lattice results for the charge and matter radii are collected in Table II. The main observation is that hadron sizes show very little quark mass dependence in the quenched case, at least for the range of quark masses considered, but increase under the effect of dynamical quarks. One should keep in mind, however, that finite volume effects on these values have not been investigated here, but could be significant at the smaller quark masses.

Despite the failure of the bag model in describing the individual radial shape of the distributions it produces reasonable results for the relative widths of the charge to the matter distribution as can be seen from Table III. Both lattice 
TABLE II. Square root of the charge $\left(r_{c h}^{2}\right)$ and matter $\left(r_{m}^{2}\right)$ radii in fm extracted from the lattice data. We use the nucleon mass to convert to physical units.

\begin{tabular}{|c|c|c|c|c|c|c|}
\hline \multicolumn{7}{|c|}{ Quenched: $\beta=6.0,16^{3} \times 32$} \\
\hline$\kappa$ & \multicolumn{2}{|c|}{0.153} & \multicolumn{2}{|c|}{0.154} & \multicolumn{2}{|c|}{0.155} \\
\hline & $\sqrt{r_{c h}^{2}}$ & $\sqrt{r_{m}^{2}}$ & $\sqrt{r_{c h}^{2}}$ & $\sqrt{r_{m}^{2}}$ & $\sqrt{r_{c h}^{2}}$ & $\sqrt{r_{m}^{2}}$ \\
\hline$\pi$ & $0.444(4)$ & $0.396(6)$ & $0.454(4)$ & $0.417(6)$ & $0.465(8)$ & $0.440(7)$ \\
\hline$\rho$ & $0.457(5)$ & $0.397(18)$ & $0.470(6)$ & $0.404(37)$ & $0.482(11)$ & - \\
\hline$n$ & $0.457(4)$ & $0.383(16)$ & $0.465(6)$ & $0.384(31)$ & $0.473(11)$ & - \\
\hline$\Delta^{+}$ & $0.464(6)$ & $0.390(19)$ & $0.469(9)$ & $0.360(76)$ & $0.447(77)$ & - \\
\hline \multicolumn{7}{|c|}{$N_{f}=2: \beta=5.6,16^{3} \times 32$} \\
\hline \multirow[t]{2}{*}{$\kappa$} & \multicolumn{4}{|c|}{0.156} & \multirow[t]{2}{*}{0.15} & \\
\hline & \multicolumn{2}{|c|}{$\sqrt{r_{c h}^{2}}$} & $\sqrt{r_{m}^{2}}$ & $\sqrt{r_{c h}^{2}}$ & & $\sqrt{r_{m}^{2}}$ \\
\hline$\pi$ & \multicolumn{2}{|c|}{$0.471(3)$} & $0.411(7)$ & \multicolumn{2}{|c|}{$0.490(3)$} & $0.439(7)$ \\
\hline$\rho$ & \multicolumn{2}{|c|}{$0.481(6)$} & $0.370(52)$ & \multicolumn{2}{|c|}{$0.510(6)$} & $0.373(121)$ \\
\hline$n$ & \multicolumn{2}{|c|}{$0.485(5)$} & $0.427(14)$ & \multicolumn{2}{|c|}{$0.509(7)$} & $0.414(57)$ \\
\hline$\Delta^{+}$ & \multicolumn{2}{|c|}{$0.489(9)$} & $0.384(55)$ & \multicolumn{2}{|c|}{$0.522(9)$} & $0.436(102)$ \\
\hline
\end{tabular}

and bag model consistently predict a broader charge than matter distribution, with a very weak mass dependence. In Table III we also give the bag-model results for the charge radius which show a stronger dependence on the quark mass as well as on the channel than the lattice results.

In Fig. 16, in addition to the bag-model results, we have also included a fit of the lattice data to the ansatz $\exp \left(-m r^{\alpha}\right)$. As it can be seen this simple ansatz provides the best description to the data. In fact, except for the pion, a good fit to all other charge and matter correlators is obtained by taking $\alpha=1$, which gives a $\chi^{2}$ per degree of freedom $<1$. In this case we can identify asymptotically the mass $m$ of the pure exponential to the mass of a propagating meson.

TABLE III. Ratio of the expectation value of $\mathbf{r}^{2}$ for the charge distribution over that for the matter distribution in quenched lattice QCD and bag model using procedure A. For $\kappa=0.155$ we give the ratio only for the pion since for the other hadrons the matter radius was too noisy. The root mean square charge radius in $\mathrm{fm}$ in the bag model using procedure $\mathrm{A}$ is also given.

\begin{tabular}{|c|c|c|c|c|c|c|}
\hline \multirow[t]{2}{*}{$\kappa$} & \multicolumn{6}{|c|}{$\left\langle r_{c h}^{2}\right\rangle /\left\langle r_{m}^{2}\right\rangle$} \\
\hline & lattice & bag & lattice & bag & lattice & bag \\
\hline$\pi$ & $1.24(3)$ & 1.35 & $1.19(2)$ & 1.36 & $1.10(2)$ & 1.37 \\
\hline$\rho$ & $1.33(7)$ & 1.35 & $1.35(17)$ & 1.36 & - & 1.37 \\
\hline$n$ & $1.40(8)$ & 1.34 & $1.45(16)$ & 1.35 & - & 1.36 \\
\hline$\Delta$ & $1.41(9)$ & 1.34 & $1.7(5)$ & 1.35 & - & 1.36 \\
\hline & \multicolumn{6}{|c|}{$\sqrt{r_{c h}^{2}}$ in the bag model $(\mathrm{fm})$} \\
\hline$\kappa$ & \multicolumn{2}{|l|}{0.153} & \multicolumn{2}{|c|}{0.154} & \multicolumn{2}{|c|}{0.155} \\
\hline$\pi$ & \multicolumn{2}{|l|}{0.383} & \multicolumn{2}{|c|}{0.439} & \multicolumn{2}{|c|}{0.466} \\
\hline$\rho$ & \multicolumn{2}{|l|}{0.422} & \multicolumn{2}{|c|}{0.492} & \multicolumn{2}{|c|}{0.537} \\
\hline$n$ & \multicolumn{2}{|l|}{0.488} & \multicolumn{2}{|c|}{0.565} & \multicolumn{2}{|c|}{0.612} \\
\hline$\Delta$ & \multicolumn{2}{|l|}{0.503} & \multicolumn{2}{|c|}{0.586} & \multicolumn{2}{|c|}{0.639} \\
\hline
\end{tabular}

To fit the matter distributions we find that we need a mass of approximately twice that required to fit the charge distributions of these hadrons. The asymptotic behavior of the density correlators can be analyzed using a tree graph approximation based on the fact that at large distances light hadrons dominate [19]. In our work the ratio of the pion to the rho mass is always larger than one-half. In this case, the analysis of Ref. [19] shows that the density correlators should decay exponentially with the mass of the rho meson, expect for the rho correlator whose exponential decay at very large distances is governed by the pion mass. A similar analysis for the matter correlator predicts asymptotically an exponential decay determined from the lightest scalar in the theory. In the continuum the lightest scalar $\left(f_{0}\right)$ is heavier than the rho by $200 \mathrm{MeV}$ and one thus expects asymptotically a faster fall off of the matter correlator as compared to the charge correlator. Unfortunately, the values we obtain for the masses, $m$, of the exponential fall off from the fits cannot be identified with the mesons of the theory. In general we find slower exponential decay than expected from the mass of the lightest meson which should dominate the asymptotic behavior. Presumably, our lattice is not large enough to probe the asymptotic behavior of the correlators. The pion correlators require $\alpha<1$, or the sum of two exponentials governing the short and long distance decays respectively. If we nevertheless use the single exponential ansatz with $\alpha=1$ also for this channel the ratio of mass values needed to fit the charge and matter density distributions is about 1.4 instead of two as for the other hadrons decreasing slightly with the quark mass. This value of $\sim 1.4$ is thus in accord with the value of $\sim 1.6$ found from the study of heavy light mesons in Ref. [13].

In the case of the pseudoscalar density the bag model predicts that the integral $\int d^{3} r C_{\gamma_{5}}^{H}(r)$ is zero. This prediction of the bag model is clearly seen in Fig. 15. It was for this reason that in Fig. 13 we chose to show the lattice quenched and unquenched results for the pseudoscalar density 
weighted by $r^{2}$. As can be seen from Fig. 13, for both the quenched and the unquenched results, the bag model prediction is worse for the pion, since the lattice correlator turns negative at a larger distance. This is not surprising since the bag model is known to be worse for the pion. For the baryons on the other hand the node of the bag model distribution coincides with that of the lattice data. In all cases the lattice data have a long tail which is not reproduced in the bag model, and which favors a non-zero integral. However a careful thermodynamic and continuum extrapolation, on larger and finer lattices, is required for this quantity, especially with Wilson fermions as used here. For the smaller quark masses noise at the tail of the distribution makes the situation even worse.

\section{CONCLUSIONS}

To check our lattice procedure we performed an analysis of the charge density distributions using various types of sources. For a lattice of temporal extent $32 a$ considered here and within our statistics we found that local and Wuppertal smeared sources give consistent results when we insert the current operator at time separation from the source of $t$ $=8 a$, which is the maximal allowed separation given the anti-periodic boundary conditions in the temporal direction. The results are also in agreement with those obtained using Dirichlet boundary conditions which allow larger time separations. Therefore, for the lattice parameters used in this study, local sources are a suitable choice for the evaluation of density distributions since they have less gauge noise than smeared ones and the temporal extent is large enough to make contributions from high excited states negligible. Wall sources are shown to filter the ground state less effectively and at the maximal time separation of $8 a$ yield results that are not in agreement with the rest. Using the lattice conserved current, which has a different $a$ dependence as compared to the continuum current, these correlators show finite lattice spacing effects near the origin.
Our main conclusion from the comparison of quenched and unquenched results for the charge, the matter and the pseudoscalar densities is that no sizable unquenching effects are observed for quark masses in the range of 300-100 MeV. The charge density distribution is, in all cases, broader than the matter density. This is what is expected if a tree level classical approximation is used to describe the asymptotic behavior of these correlators. However the masses of the propagating lightest mesons which determine the exponential fall off cannot be identified with the mesons of the theory. To observe the true asymptotic behavior larger lattices are needed. For the pion, the lattice results are in agreement with the experimentally extracted ratio of the charge to the matter radius of 1.15(9) [3]. For baryons, the lattice indicates a charge radius about $20 \%$ larger than the matter radius. This effect is well reproduced by the bag model. However the bag model does a poor job in the description of the radial dependence, especially in the case of the mesons. Instead, the charge and the matter distributions are well described by the simple ansatz $\exp \left(-m r^{\alpha}\right)$, with $\alpha=1$ except for the pion. One prediction of the bag model is that the volume integral of the pseudoscalar density is zero. Lattice data for both the quenched and the unquenched theory favor a negative value. A larger lattice is required to settle this issue.

The deformation seen in the rho charge distribution is absent in the matter distribution, both in the quenched and the unquenched theory. This observation suggests a relativistic origin for the deformation. This important issue deserves a more extended study, with lighter quarks and larger volumes.

\section{ACKNOWLEDGMENTS}

The $S U(3) 16^{3} \times 32$ quenched lattice configurations were obtained from the Gauge Connection archive [7]. We thank the SESAM Collaboration for giving us access to their dynamical lattice configurations. A.T. acknowledges the Levendis Foundation.
[1] A. Kohama, R. Seki, A. Arima, and Sh. Yamaji, nucl-th/0211025.

[2] C.W. Akerlof et al., Phys. Rev. D 14, 2864 (1976).

[3] T.T. Chou, Phys. Rev. D 11, 3145 (1975).

[4] C. Alexandrou, Ph. de Forcrand, and A. Tsapalis, Phys. Rev. D 66, 094503 (2002).

[5] C. Alexandrou, Ph. de Forcrand, and A. Tsapalis, Nucl. Phys. B (Proc. Suppl.) 119, 422 (2003).

[6] C. Alexandrou, Ph. de Forcrand, and A. Tsapalis, Nucl. Phys. A 721, 907 (2003).

[7] http://qcd.nersc.gov

[8] SESAM Collaboration, N. Eicker et al., Phys. Rev. D 59, 014509 (1999).

[9] QCD-TARO Collaboration, S. Choe et al., Nucl. Phys. B (Proc. Suppl.) 106, 361 (2002); J. High Energy Phys. 08, 022 (2003).

[10] G.S. Bali, C. Schlichter, and K. Schilling, Phys. Rev. D 51, 5165 (1995).
[11] Y. Iwasaki et al., Phys. Rev. D 53, 6443 (1996).

[12] T. Bhattacharya, R. Gupta, G. Kilcup, and S. Sharpe, Phys. Rev. D 53, 6486 (1996).

[13] A.M. Green, J. Koponen, and P. Pennanen, Eur. Phys. J. C 28, 79 (2003).

[14] R. Gupta, D. Daniel, and J. Grandy, Phys. Rev. D 48, 3330 (1993).

[15] A.J. Buchmann and E.M. Henley, Phys. Rev. C 63, 015202 (2001).

[16] A.J. Buchmann, E. Hernández, and A. Faessler, Phys. Rev. C 55, 448 (1997).

[17] T. DeGrand, R.L. Jaffe, K. Johnson, and J. Kiskis, Phys. Rev. D 12, 2060 (1975).

[18] M. Lissia, M.-C. Chu, J.W. Negele, and J.M. Grandy, Nucl. Phys. A555, 272 (1993).

[19] J.W. Negele, M. Burkardt, and J.M. Grandy, Ann. Phys. (N.Y.) 238, 441 (1995). 